\title{
Flexor Digitorum Longus Muscle - an Unusual Presentation; Anatomy and Clinical Significance, A case report
}

\author{
Dr. Renu Gupta ${ }^{1} \&$ Dr. Brijendra Singh ${ }^{2}$ \\ 1. Assistant Professor 2. Additional Professor, Department of Anatomy, All India Institute of Medical Sciences, \\ Jodhpur-342005 Rajasthan, INDIA.
}

\begin{abstract}
Understanding muscle architecture of the foot may assist in the design of surgical procedures such as tendon transfer, biomechanical modeling of the foot, prosthesis design, and analysis of foot function. This paper primarily focuses on the variation of Flexor Digitorum Longus and Brevis muscles of foot. Sixty inferior extremities were dissected. While dissecting a body of adult female we observed a small muscle belly taking origin from the medial and lateral processes of the calcaneal tuberosity and get inserted in the distal phalanx of the little toe of right foot. The 4th tendon of the Flexor Digitorum Brevis muscle was absent and the respective tendon of the Flexor Digitorum Longus muscle to the little toe was atrophied in same foot. This additional muscle belly is pierced by one of branch of lateral planter nerve. The importance of such variations is to be kept in mind during radio-diagnostic procedures and surgical interventions.

Flexor digitorum longus muscle - unusual presentation, its anatomy and clinical significance
\end{abstract}

\section{Introduction}

The posterior compartment of leg is the largest at commencement but it diminishes markedly as it approaches the ankle. Flexor digitorum longus is thin and pointed proximally and gradually widens as it descends in the posterior compartment of leg. It arises from the posterior surface of the tibia medial to tibialis posterior, just below the soleal line, within seven or eight $\mathrm{cm}$ of the distal end of bone; it also arises from the fascia covering tibialis posterior. The muscle ends in a tendon that extends along almost the whole of its posterior surface. It passes deep to flexor retinaculum in the tarsal tunnel and enters sole of the foot. In the sole, the flexor digitorum longus muscle passes superficial to flexor hallucis longus tendon, crosses it from medial to lateral side, divides into four tendons which are inserted into the plantar surface of the base of distal phalanx of the lateral four toes (1).

The tendons of flexor digitorum accessorius insert into the flexor digitorum longus tendons of the second, third and fourth toes. The long flexor tendons of the lateral four toes are attached to the plantar surfaces of the bases of their distal phalanges. It is innervated by the tibial nerve.

Multiple accessory, supernumerary and variant muscles have been described in the anatomical, radiological and surgical literature. Here, we present a case report of a variant muscle originating from the tendon of flexor digitorum longus muscle in sole.

\section{Case Report}

We were dissected 60 inferior extremities and a tendon variation of FDL and FDB muscles were observed in an adult female formalin-fixed cadaver at the All India Institue of Medical Sciences, Anatomy, Dissection Laboratory during dissection for undergraduate teaching and demonstration. Right and left soles were dissected in a standard manner for study and comparison.

We observed variations in the flexor digitorum brevis and longus muscles of right foot. In the right foot, the 4th tendon of the flexor digitorum brevis muscle was absent (fig 1) and the respective tendon of the flexor digitorum longus muscle to the little toe was atrophied. In the left foot, the 4th tendons of both the flexor digitorum brevis and longus muscles to the little toe were present. The lacking deep flexor tendon to the little toe in the right foot was replaced by an isolated flexor muscle originating from the medial and lateral processes of the calcaneal tuberosity. The tendon of isolated flexor muscle inserted in the distal phalanx of the little toe. This muscle belly is pierced by one of branch of lateral planter nerve which is one of terminal branch of tibial nerve (fig 2).

\section{Discussion}

FDB and FDL along with the other intrinsic muscles of the foot help to maintain the longitudinal arches of the foot. Many authors in various classic Anatomy text books have mentioned that FDB gives four tendons to the lateral four toes. Variations in FDB have been reported to occur in 63.0\% of all limbs (2). Though absence of FDB tendon to the fifth toe was reported by Chaney et al (3). and Sarrafian(4), in the present study we observed that FDB tendon to the fifth toe was entirely missing in right foot but normal on left side. The 
usage of the fifth toe in humans is minimal. According to Darwin's disuse theory, therefore, FDB tendon to the fifth toe may be undergoing phylogenetic variation. This is supported by Reeser et al. (5) in their electromyographic study of human foot which showed that FDB is not preferentially recruited over flexor digitorum longus for any normal posture or locomotion.

Asomugha et al (6). have reported accessory flexor of the fifth toe. This muscle originated from the tendon of tibialis posterior and inserted into the middle phalanx of the fifth toe. In present study we observed an isolated flexor muscle belly originating from the medial and lateral processes of the calcaneal tuberosity and get inserted in the distal phalanx of the little toe.

Accessory muscles of the ankle are typically asymptomatic but can cause pain, compressive neuropathy, compartment syndrome or rigid hindfoot deformities, and can also mimic soft tissue tumors. From the above literature review it is quite evident that flexor digitorum longus is an important muscle as its functional and clinical significance is important not only for the anatomist and clinicians but also for the pathologists and sports personals. Its variations are not very common. Knowledge of these accessory muscles, their specific location and characteristic CT and MRI appearance assist in avoiding wrong diagnosis, guiding treatment and directing surgical options. Awareness of the variations of FDB is important in the studies related to hominid evolution.

\section{References:}

[1] Standring S, Ellis H, Healy JC, Johnson D, Williams A, Collins P, Wigley C. Gray's Anatomy. 39th Ed., Churchill Livingstone, New York. 2005; 1500 .

[2] Bergman RA, Thompson SA, Afifi AK, Saadeh FA. Compendium of Human Anatomic Variations. Baltimore: Urban and Schwarzenberg 1988.

[3] Chaney DM, Lee MS, Khan MA, Krueger WA, Mandracchia VJ, Yoho RM. 1996. Study of ten anatomical variants of the foot and ankle. J Amer Podiatr Med Assoc 1996; 86: 532-7.

[4] Sarrafian SK. Anatomy of the Foot and Ankle. Philadelphia: Lippincott Williams and Wilkins 1983.

[5] Reeser LA, Susman RL, Stern JT Jr. Electromyographic studies of the human foot: experimental approaches to hominid evolution. Foot Ankle 1983; 3: 391-407.

[6] Asomugha AL, Chukwuanukwu TO, Nwajagu GI, Ukoha U. An accessory flexor of the fifth toe. Niger J Clin Pract 2005; 8: 130-2.

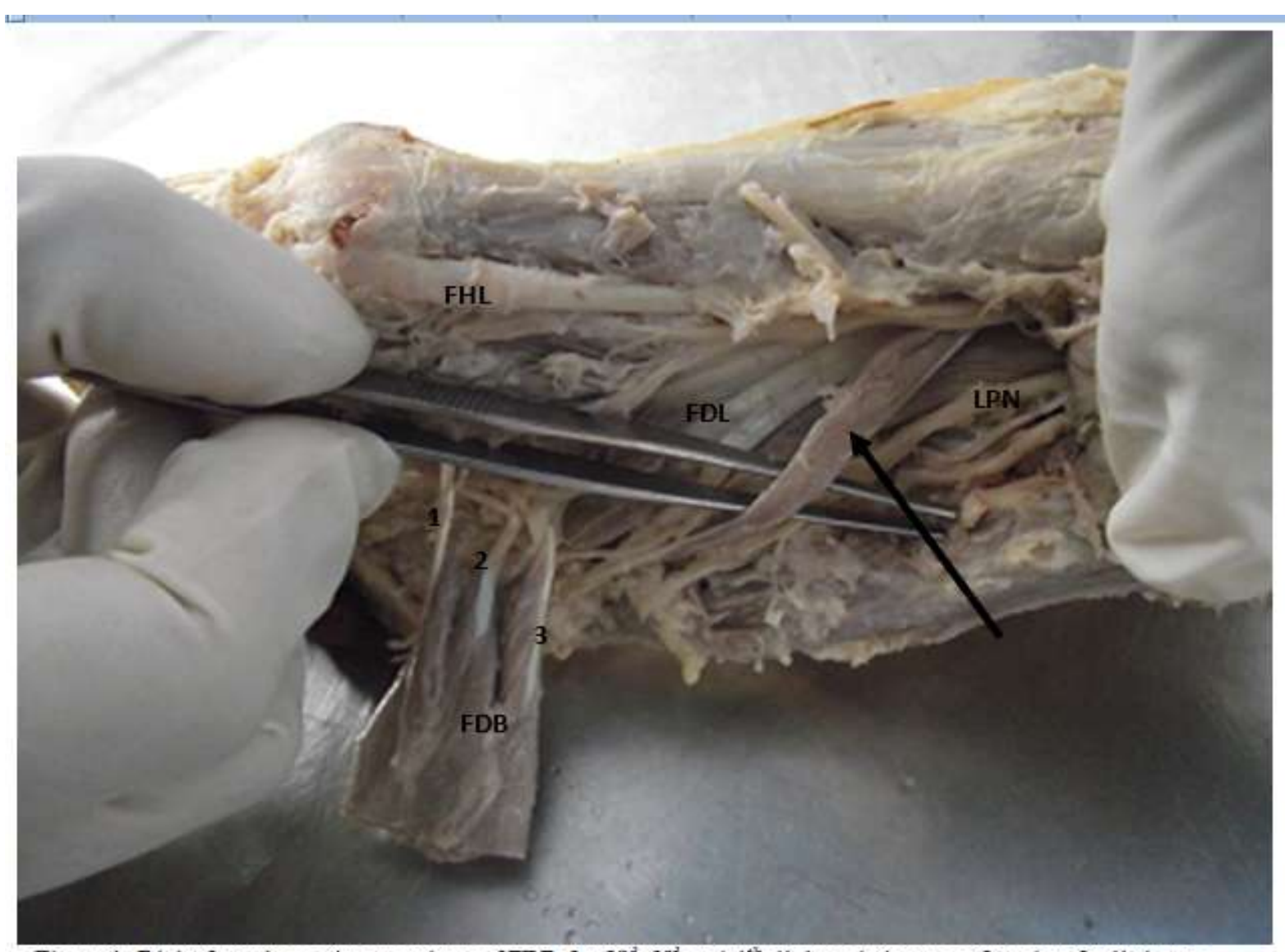

Figure 1: Right foot shows three tendons of FDB for $2^{\text {nd }}, 3^{\text {rd }}$ and $4^{\text {th }}$ digit and absence of tendon for little toe.

Tendons of FDL also seen along with additional flexor muscle belly (arrow indicates) arises from medial and lateral process of Calcaneum. Flexor. Hallusis Longus (FHL) and Lateral Planter Nerve (LPN) also shown in figure. 


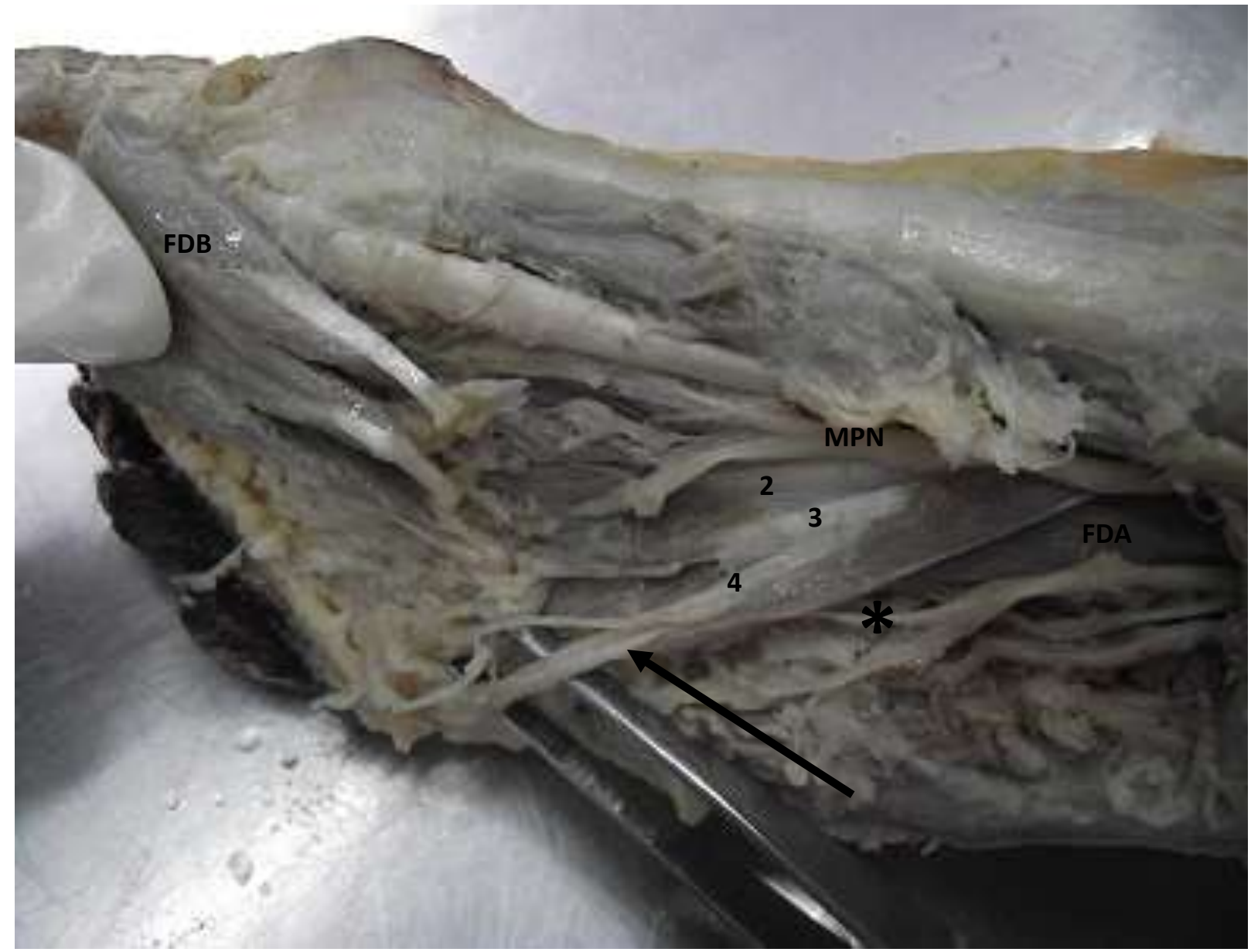

Figure 2: Right foot shows $2^{\text {nd }}, 3^{\text {rd }}$ and $4^{\text {th }}$ tendon of FDL for lateral three toes. Tendon for little toe is atrophied which is supplemented by tendon of additional belly (arrow indicate). This belly pierced by branch of tibial nerve (asterix shows). Medial planter nerve (MPN) and belly of Flexor Digitorum Accessorius (FDA) also seen in figure. 